\title{
Morbidity Profile and Causes of Mortality in Type 2 Diabetes Patients: Data from a Tertiary Teaching Hospital from Eastern India
}

\author{
Soumya Ranjan Behera', Manoranjan Behera ${ }^{2 *}$, Sidhartha Das ${ }^{2}$, Bhabani Prasad Panda ${ }^{3}$, \\ Saroj Kumar Tripathy2, Purna Chandra Dash² \\ ${ }^{1}$ Department of Medicine, VSS Institute of Medical Sciences and Research, Burla, Sambalpur, Odisha, India \\ ${ }^{2}$ PG Department of Medicine, Faculty of Medicine, Sriram Chandra Bhanja Medical College, Cuttack, Odisha, India \\ ${ }^{3}$ PG Department of Medicine, Sriram Chandra Bhanja Medical College, Cuttack, Odisha, India \\ Email: *doctor_manoranjan@rediffmail.com
}

How to cite this paper: Behera, S.R., Behera, M., Das, S., Panda, B.P., Tripathy, S.K. and Dash, P.C. (2017) Morbidity Profile and Causes of Mortality in Type $2 \mathrm{Di}$ abetes Patients: Data from a Tertiary Teaching Hospital from Eastern India. Journal of Diabetes Mellitus, 7, 195-211. https://doi.org/10.4236/jdm.2017.73016

Received: July 27, 2017

Accepted: August 21, 2017

Published: August 24, 2017

Copyright (ङ 2017 by authors and Scientific Research Publishing Inc. This work is licensed under the Creative Commons Attribution International License (CC BY 4.0).

http://creativecommons.org/licenses/by/4.0/

\section{Abstract}

Background: Data regarding the prevalence of morbidity and mortality in patients of Type 2 Diabetes Mellitus (T2DM) is scanty in India. Objectives: To determine the prevalence of micro and macro vascular complications, acute metabolic complications, infections, Non Alcoholic Fatty Liver Disease (NAFLD) and cause of mortality in T2DM patients admitted to a tertiary care teaching hospital in Eastern India. Material and Methods: This was a hospital-based prospective study evaluating $150 \mathrm{~T} 2 \mathrm{DM}$ patients admitted to a tertiary care institution in Eastern India. Diagnosis of micro and macro vascular complications, infections and NAFLD was made using standard protocols. In case of death, the most probable cause was noted. Results: Out of 150 patients, $14.7 \%$ of patients were newly diagnosed T2DM and out of them $41 \%$ of patients had vascular complications and $54.5 \%$ had infections. Of the total patients, $56 \%$ had nephropathy, 20\% neuropathy, $17.3 \%$ retinopathy, $31.3 \%$ CVD, $11.3 \%$ CAD, $4.6 \%$ acute metabolic complications, $44 \%$ infections and $16.6 \%$ had NAFLD respectively. Macrovascular events occured earlier than microvascular complications. Multiple logistic regression analysis showed strong association of age, duration of diabetes, serum cholesterol, triglyceride, LDL-C with retinopathy (Regression coefficient $\beta$ : $-0.1086807,0.4127152,-0.0513393$, $0.0146429,0.0587475 ; \mathrm{p}<0.05,<0.001,<0.05,<0.05,<0.05$ respectively), while only duration of diabetes was strongly associated with nephropathy and neuropathy (Regression coefficient $\beta: 0.2538751,0.2261636 ; \mathrm{p}<0.001$ for each). Increasing age was associated with CAD (Regression coefficient $\beta$ : 0.055392; $\mathrm{p}<0.05$ ) and FBG was a risk factor for CVD (Regression coefficient $\beta: 0.0055014 ; \mathrm{p}<0.05) .18 .6 \%$ patients died due to diabetes related complications. Cardiovascular (CV)-related deaths $(\mathrm{CVD}+\mathrm{CAD})$ were most common 
cause (51.5\%: CVD 36.4\%, CAD 15.1\%) to be followed by infections $(27.3 \%)$ and then chronic kidney disease (12.1\%). Conclusions: This study highlights the high prevalence of vascular complications and infections in T2DM patients of Eastern India. CV-related deaths were principal causes of death, similar to that in developed world.

\section{Keywords}

Type 2 Diabetes Mellitus, Vascular Complications, Infections, NAFLD, Mortality

\section{Introduction}

Prevalence of diabetes mellitus (DM) is on an increase in India and Type 2 DM (T2DM) is the most prevalent form of DM seen in India, which constitutes more than $95 \%$ of the diabetes population. It has been observed in many studies that over $50 \%$ of patients with DM in India have a poor glycemic control and a substantial proportion amongst these also have diabetes-related complications [1]. According to the Chennai Urban Population Study (CUPS), the prevalence of coronary artery disease (CAD) was $21.4 \%$ (CUPS No 5) [2] and peripheral vascular disease (PVD) was 6.3\% among patients of DM in South India [3]. Another study from North Delhi Diabetes centre comprising 720 T2DM patients, retinopathy was found in $21.2 \%$, microalbuminuria in $41 \%$, peripheral neuropathy in $15.3 \%, \mathrm{CAD}$ in $7 \%$ and PVD in $7.4 \%$ of patients [1].

T2DM patients are susceptible to develop many acute and chronic infections primarily due to altered host immunity [4]. Urinary tract infection (UTI) is the most common infection and is a common cause for hospital admission in subjects of T2DM. Subjects with T2DM are at a three-fold higher risk of developing tuberculosis (TB) and it accounts for $15 \%$ of all TB and $21 \%$ of smear positive TB Worldwide [5].

According to the CUPS-16, the overall mortality was nearly 2 -fold higher in diabetic subjects compared to non-diabetic cohorts (18.9 vs. 5.3 per thousand person years, $\mathrm{P}=0.004$ ) [6]. The study also reported that the prevalence of mortality due to cardio vascular disease (diabetic subjects: $52.9 \%$, non-diabetic subjects: $24.2 \%, \mathrm{P}=0.042$ ) followed by renal disease (diabetic subjects: $23.5 \%$, non-diabetic subjects: $6.1 \%, \mathrm{P}=0.072$ ) was higher among diabetic subjects compared to non-diabetic counterparts [6]. A study from a tertiary care hospital of Odisha in 1991 had revealed the mortality pattern in DM patients as follows: chronic kidney disease (CKD) 35\%, infection 33.7\%, cerebrovascular disease (CVD) $32.5 \%$, CAD $30 \%$ and diabetic coma $20.2 \%$ respectively [7].

Since then, there has been no systematic study on burden of morbidity and mortality in T2 DM patients in this part of the country. Hence, the present study was undertaken to determine the prevalence of microvascular complications, macrovascular complications, acute metabolic complications, other complications like infection, Non-Alcoholic Fatty Liver Disease (NAFLD) and to evaluate 
the causes of mortality in patients of T2DM in this part of the country.

\section{Material and Methods}

This study was a prospective hospital based study conducted in the postgraduate department of Medicine, S.C.B. Medical College and Hospital, Cuttack, Odisha, India. One hundred and fifty cases of T2 DM patients admitted consecutively to the first unit of this department from August 2014 to November 2015 of both gender and age group $\geq 30$ year were enrolled for the study. Patients with other forms of DM, history of chronic alcoholism or smoking, HIV/AIDS, those on immunosuppressive therapy or on chronic use of drugs known to cause vascular or neurological complications were excluded from this study. Institutional ethical committee clearance was duly obtained.

Each patient underwent complete clinical examination with detailed history obtained during the examination. Details regarding age, sex, urban or rural, socio-economic status, duration of diabetes and treatment history of diabetes were recorded for all the patients. DM was diagnosed according to WHO criteria [8]. Blood glucose level estimation was done by glucose oxidase method using a standard kit supplied by Acutex Biochemical Pvt. Ltd. (Mumbai, India). Glycosylated haemoglobin $\left(\mathrm{HbA}_{1} \mathrm{C}\right)$ was measured by ion-exchange chromatography method. Lipid profile, liver function test, blood urea, serum creatinine, and serum electrolytes were done by auto analyser (TBA $120 \mathrm{FR}$, TOSHIBA) using specific kits. Serum cholesterol was estimated using a standard kit (Enzokit) supplied by Ranbaxy Fine Chemicals Ltd. Diagnostic Centre; serum triglyceride (TG) was estimated using a standard kit supplied by Chemelex SA, Barcelona; serum low-density lipoprotein cholesterol (LDL-C) was estimated using a standard kit supplied by Agappe Diagnostics Ltd. (Kerala, India); serum high-density lipoprotein cholesterol (HDL-C) was estimated using a standard kit supplied by Transasia Biomedicals Ltd. (Daman, India) and the Boehringer Mannheim photometer 5010 (Birkenfeld, Germany). Very low-density lipoprotein cholesterol (VLDL-C) was estimated by dividing the TG by 5 .

The selected patients were evaluated for the presence of microvascular complications (retinopathy, nephropathy, and neuropathy), macrovascular complications (CAD, CVD, and PVD), acute metabolic complications, infections and NAFLD by clinical examination and relevant investigations as enumerated below. The cause of mortality of those patients who died inpatient was also recorded.

Direct and indirect fundoscopy was used to make the diagnosis and grading of retinopathy [9]. Nephropathy was diagnosed based on 24 hour urine albumin excretion rate (AER), done by immunoturbidimetry method. Incipient nephropathy was diagnosed by microalbuminuria (30 - $300 \mathrm{mg} / 24$ hours) and overt nephropathy was established by macroalbuminuria ( $>300 \mathrm{mg} / 24$ hours), abnormality in renal function, raised serum creatinine and estimation of glomerular filtration rate (GFR: $>60 \mathrm{~mL} / \mathrm{min}$, CKD stage $1+2 ; 30-60 \mathrm{~mL} / \mathrm{min}$, CKD stage 
$3 ;<30 \mathrm{~mL} / \mathrm{min}$, CKD stage $4+5$ ) [10]. Neuropathy was diagnosed by history of paresthesia, numbness and tingling sensation and confirmed by touch sensation with 10-g monofilament pressure sensation at the distal plantar aspect of both great toes and metatarsal points, vibration sensation with $128 \mathrm{~Hz}$ tuning fork, ankle reflexes and nerve conduction studies [11].

$\mathrm{CAD}$ was diagnosed from history of angina or myocardial infarction documented by previous medical records or by ECG changes of ST-segment, Q-wave, or T-wave (Minnesota code) suggestive of CAD [2] as well as 2D-echocardiography. CVD was diagnosed from the history of neurological deficit, clinical examination and CT scan/MRI of brain. Diabetic keto acidosis (DKA) and hyperglycemic hyperosmolar state (HHS) were diagnosed by standard diagnostic criteria. NAFLD was diagnosed by ultrasonography showing evidences of fatty liver [12].

Other relevant tests done were total leucocyte count, differential count, hemoglobin, urine routine and microscopic examination. Urine for ketone bodies/ culture and sensitivity test, blood culture and sensitivity test, arterial blood gas analysis (ABG), and chest X-Ray (postero-anterior view) were done whenever required.

Statistical analysis was done using SPSS statistical package version 20.0. Quantitative variables were described as mean $+/$-standard deviation (SD) unless otherwise indicated. Qualitative variables were described by percentage. Multiple logistic regression analysis with stepwise additions of variables was performed to assess their association with each of the complication studied. For all statistical tests, $\mathrm{p}$ value $<0.05$ was considered significant.

\section{Results}

Our study is a hospital based study and reflects the prevalence of diabetic complications in the in-patient setting.

Of the total 150 recruited patients with T2DM, majority were male $(68 \%, \mathrm{n}=$ $102)$, female $(32 \%, \mathrm{n}=48), \mathrm{M}: \mathrm{F}(2.1: 1)$. The age of the patients studied ranged between 30 - 90 years with a mean age of $60.46( \pm 12.6)$ years. The importance of age on prevalence of diabetes shows that majority of patients were from age group of 50 - 70 years (53.3\%). Family history of diabetes was present in one third of patients $(33.3 \%)$. The patients on antidiabetic medication were $85.3 \%$. $14.7 \%(\mathrm{n}=22)$ patients were newly diagnosed with T2 DM out of which $40.9 \%$ ( $\mathrm{n}=09$ ) patients were diagnosed to have vascular complications (nephropathy, retinopathy and CAD in $09 \%(\mathrm{n}=02)$ each, neuropathy in $4.5 \%(\mathrm{n}=01)$ cases, CVD in $31.8 \%(n=07)$ cases and $54.5 \%(n=12)$ had infections as complications mandating hospitalisation.

The prevalence of diabetes specific microvascular and macrovascular complications are shown in Table 1. Prevalence of microvascular complications was more than macrovascular complications. Among the microvascular complications, nephropathy was the most common form followed by neuropathy and retinopathy. In patients having diabetic retinopathy, majority (96\%) were having nonproliferative diabetic retinopathy (NPDR). CVD was the most common ma- 
crovascular complication to be followed by CAD and there were no case with evidence of PVD. Amongst CVD, 81\% had cerebral infarction and 19\% had intracerebral haemorrhage (ICH). Out of 150 patients, 24 patients (16\%) did not have any vascular complications. The relationship of mean duration of diabetes and different vascular complications were as follows: retinopathy $10.23( \pm 6.34)$ years, neuropathy $9.36( \pm 7.11)$ years, nephropathy $7.89( \pm 5.2)$ years, CAD 7.88 $( \pm 5.21)$ years, and CVD $5.87( \pm 5.06)$ years as shown in Table 2. It signifies that macrovascular events occured earlier than microvascular complications. The prevalence of risk factors for macrovascular disease was as follows; Hypertension $46.6 \%$, obesity $44 \%$, and dyslipidemia in $25.3 \%$ of cases.

Relationship of different risk factors with all vascular complications is shown in Table 2. Blood pressure, total cholesterol and LDL-C were higher in patients having CVD, where as age and TG were higher in patients of CAD than patients

Table 1. Prevalence of vascular complications in Type 2 DM.

\begin{tabular}{cccc}
\hline Complications & Male (\%) & Female (\%) & Overall (\%) (N:150) \\
\hline Microvascular & & & 56 \\
Nephropathy & 53.9 & 60.4 & $(\mathrm{~N}: 84)$ \\
Neuropathy & $(\mathrm{N}: 55)$ & 25 & 20 \\
& 17.6 & $(\mathrm{~N}: 12)$ & $(\mathrm{N}: 30)$ \\
Retinopathy & $(\mathrm{N}: 18)$ & 29.1 & 17.3 \\
& 11.7 & $(\mathrm{~N}: 14)$ & $(\mathrm{N}: 26)$ \\
Macrovascular & $(\mathrm{N}: 12)$ & & 31.33 \\
Cerebrovascular & & 33.33 & $(\mathrm{~N}: 47)$ \\
Disease (CVD) & 30.39 & $(\mathrm{~N}: 16)$ & 11.33 \\
Coronary Artery & $(\mathrm{N}: 31)$ & 6.25 & $(\mathrm{~N}: 17)$ \\
Disease (CAD) & 13.72 & $(\mathrm{~N}: 03)$ & \\
\hline
\end{tabular}

Table 2. Relationship of different risk factors with complications.

\begin{tabular}{cccccc}
\hline PARAMETERS & Retinopathy & Neuropathy & Nephropathy & CAD & CVD \\
\hline Age (Years) & $58.92 \pm 14.07$ & $60.93 \pm 14.60$ & $62.12 \pm 12.17$ & $66.94 \pm 11.80$ & $61.91 \pm 11.71$ \\
$\begin{array}{c}\text { Duration of DM } \\
\text { (Years) }\end{array}$ & $10.23 \pm 6.34$ & $9.36 \pm 7.11$ & $7.89 \pm 5.2$ & $7.88 \pm 5.21$ & $5.87 \pm 5.06$ \\
SBP (mm Hg) & $141.69 \pm 26.63$ & $141.13 \pm 35.30$ & $144.55 \pm 31.42$ & $138.2 \pm 30.2$ & $166.85 \pm 33.28$ \\
DBP (mm Hg) & $81.15 \pm 7.4$ & $79.4 \pm 8.5$ & $81.01 \pm 10.02$ & $78.23 \pm 9.1$ & $88.38 \pm 5.3$ \\
BMI (kg/m²) & $24.01 \pm 2.96$ & $24.44 \pm 4.19$ & $23.97 \pm 3.07$ & $24.616 \pm 2.25$ & $24.22 \pm 2.87$ \\
HbA1c (\%) & $8.74 \pm 1.93$ & $8.40 \pm 1.61$ & $8.27 \pm 1.79$ & $8.386 \pm 1.32$ & $8.46 \pm 1.78$ \\
Total cholesterol & $149.38 \pm 57.01$ & $168.67 \pm 66.18$ & $151.15 \pm 57.39$ & $159.24 \pm 66.1$ & $178.40 \pm 60.18$ \\
$(\mathrm{mg} / \mathrm{dL})$ & $38.31 \pm 12.18$ & $38.60 \pm 11.29$ & $36.49 \pm 10.59$ & $40.71 \pm 11.29$ & $42.57 \pm 11.21$ \\
HDL-C (mg/dL) & & & & & \\
LDL-C (mg/dL) & $90.15 \pm 37.76$ & $91.67 \pm 47.57$ & $84.49 \pm 40.77$ & $90.71 \pm 42.81$ & $105.30 \pm 48.40$ \\
TG (mg/dL) & $140.03 \pm 13.2$ & $134.46 \pm 11.3$ & $128.24 \pm 12.3$ & $146.11 \pm 11.4$ & $135.85 \pm 10.3$ \\
\hline
\end{tabular}

(Abbreviations: CAD, coronary artery disease; CVD, cerebrovascular disease; DM, diabetes mellitus; SBP, systolic blood pressure; DBP, diastolic blood pressure; BMI, body mass index; HbAlc, glycosylated hemoglobin; HDL-C, high density lipoprotein-cholesterol; LDL-C, low density lipoprotein-cholesterol; TG, triglyceride). 
having other complications. HDL-C level were either normal or higher in subjects suffering from CVD or CAD. Similarly mean $\mathrm{HbA}_{1} \mathrm{C}$ was highest in the retinopathy group.

Table 3 shows the profile of other complications. Infection related complications were found in $44 \%$ cases. The profile of infections is shown in Table 4. UTI followed by pneumonia and septicemia were most common in these patients. E. coli was the most common (62\%) pathogen isolated from the urine sample followed by $S$. aureus in $14.3 \%$ cases. No growth was found in $19.4 \%$ cases.

Considering the all cause mortality, 22\% patients died due to different complications. CVD was the most common cause of death followed by infection, CAD, CKD and HHS in descending order. Hence, cardio-vascular (CV) related death (CVD and CAD) was the most common cause of death as shown in Table 5 .

Table 6 shows the association of age, duration of DM, systolic and diastolic blood pressure, fasting blood glucose (FBG), HbA1C, BMI, serum cholesterol, serum TG, serum HDL-C, serum LDL-C and serum VLDL-C with microvascular complications. Among these factors age, duration of diabetes, serum cholesterol, serum TG and serum LDL-C showed strongest association with retinopa-

Table 3. Other complications.

\begin{tabular}{cc}
\hline Other complications & Overall (\%) \\
Hypoglycemia & $(\mathrm{N}: 150)$ \\
\hline Hyperglycemic Hyperosmolar State (HHS) & 5.3 \\
Diabetic Ketoacidosis (DKA) & $(\mathrm{N}: 08)$ \\
Infections & 4.0 \\
& $(\mathrm{~N}: 06)$ \\
Non Alcoholic Fatty Liver Disease & 0.6 \\
& $(\mathrm{~N}: 01)$ \\
& 44 \\
& $(\mathrm{~N}: 66)$ \\
\end{tabular}

Table 4. Profile of infections in Type 2 DM.

\begin{tabular}{cc}
\hline Types of Infections & $\begin{array}{c}\text { Overall \% } \\
(\mathrm{N}: 66)\end{array}$ \\
\hline Urinary Tract Infection & $31.8(\mathrm{~N}: 21)$ \\
Pneumonia & $30.3(\mathrm{~N}: 20)$ \\
Septicemia & $30.3(\mathrm{~N}: 20)$ \\
Malaria & $06(\mathrm{~N}: 04)$ \\
Enteric fever & $06(\mathrm{~N}: 04)$ \\
Diabetic Foot & $4.5(\mathrm{~N}: 03)$ \\
Tuberculosis & $4.5(\mathrm{~N}: 03)$ \\
Encephalitis & $4.5(\mathrm{~N}: 03)$ \\
Dengue & $03(\mathrm{~N}: 02)$ \\
\hline
\end{tabular}


Table 5. Causes of mortality $(\mathrm{n}=33)$.

\begin{tabular}{cc}
\hline Causes & Percentage (No of patients) \\
\hline CVD & $36.4(\mathrm{n}=12)$ \\
Infection & $27.3(\mathrm{n}=9)$ \\
CAD & $15.16(\mathrm{n}=5)$ \\
CKD & $12.1(\mathrm{n}=4)$ \\
HHS & $9.0(\mathrm{n}=3)$ \\
\hline
\end{tabular}

(Abbreviations: CVD, cerebrovascular disease; CAD. coronary artery disease; CKD, chronic kidney disease; HHS, hyperglycemic hyperosmolar state).

Table 6. Results of multiple logistic regression analysis showing association of various risk factors with microvascular complications. (a) Diabetic nephropathy; (b) Diabetic neuropathy; (c) Diabetic retinopathy.

(a)

\begin{tabular}{ccccc}
\hline Variable & Odds Ratio & $95 \% \mathrm{Cl}$ & Regression coefficient $\beta$ & P value \\
\hline Age (Years) & 0.997489 & $0.9615452-1.034777$ & -0.0025141 & 0.893 \\
$\begin{array}{c}\text { Duration of DM } \\
\text { (Years) }\end{array}$ & 1.289011 & $1.151751-1.442628$ & 0.2538751 & 0.000 \\
SBP (mm Hg) & 0.9981147 & $0.9712355-1.025738$ & -0.0018871 & 0.892 \\
DBP (mm Hg) & 0.9785502 & $0.9095401-1.052796$ & -0.0216832 & 0.561 \\
BMI $\left(\mathrm{Kg} / \mathrm{m}^{2}\right)$ & 1.011192 & $0.8888238-1.150408$ & 0.0111301 & 0.866 \\
FBG $(\mathrm{mg} / \mathrm{dL})$ & 1.000417 & $0.9958869-1.004968$ & 0.0004168 & 0.857 \\
HbAlc $(\%)$ & 1.062085 & $0.835399-1.350238$ & 0.0602341 & 0.623 \\
Cholesterol (mg/dL) & 1.004075 & $0.9860692-1.02241$ & 0.0040668 & 0.660 \\
Triglyceride (mg/dL) & 0.9918294 & $0.9827685-1000974$ & -0.0082041 & 0.080 \\
HDL-C (mg/dL) & 0.9496184 & $0.900919-1.00095$ & -0.0516951 & 0.054 \\
LDL-C (mg/dL) & 0.9987817 & $0.9783527-1.019637$ & -0.0012191 & 0.908 \\
VLDL-C (mg/dL) & 1.04021 & $0.9797455-1.104405$ & 0.0394223 & 0.197 \\
\hline
\end{tabular}

(b)

\begin{tabular}{ccccc}
\hline Variable & Odds Ratio & $95 \% \mathrm{Cl}$ & Regression coefficient $\beta$ & P value \\
\hline $\begin{array}{c}\text { Age (Years) } \\
\begin{array}{c}\text { Duration of DM } \\
\text { (Years) }\end{array}\end{array}$ & 0.9662639 & $0.9220365-1.012613$ & -0.0343183 & 0.151 \\
SBP (mm Hg) & 0.9927321 & $0.9617348-1.024738$ & -0.0072944 & 0.000 \\
DBP (mm Hg) & 0.9937687 & $0.915065-1.079242$ & -0.0062508 & 0.652 \\
BMI (kg/m $)$ & 1.043847 & $0.9090641-1.198614$ & 0.0429131 & 0.882 \\
FBG (mg/dL) & 1.004511 & $0.9991358-1.009916$ & 0.0045012 & 0.543 \\
HbAlc (\%) & 0.9856609 & $0.7349496-1.321897$ & -0.0144429 & 0.100 \\
Cholesterol (mg/dL) & 1.00659 & $0.9882576-1.025262$ & 0.0065683 & 0.923 \\
Triglyceride (mg/dL) & 0.9942332 & $0.9829178-1.005679$ & -0.0057835 & 0.484 \\
HDL-C (mg/dL) & 1.004526 & $0.9471536-1.065374$ & 0.0045162 & 0.322 \\
LDL-C (mg/dL) & 0.9956035 & $0.9733089-1.018409$ & -0.0044062 & 0.880 \\
VLDL-C (mg/dL) & 1.033067 & $0.9600939-1.111587$ & 0.0325322 & 0.703 \\
\hline
\end{tabular}


(c)

\begin{tabular}{ccccc}
\hline Variable & Odds Ratio & $95 \% \mathrm{Cl}$ & Regression coefficient $\beta$ & P value \\
\hline $\begin{array}{c}\text { Age (Years) } \\
\text { Duration of DM } \\
\text { (Years) }\end{array}$ & 0.8970168 & $0.8401426-.9577411$ & -0.1086807 & 0.001 \\
SBP (mm Hg) & 0.969123 & $0.9328745-1.006781$ & -0.0313633 & 0.107 \\
DBP (mm Hg) & 1.098773 & $0.9853866-1.225207$ & 0.0941942 & 0.090 \\
BMI (kg/m $)$ & 0.9680751 & $1.020503-1.10208$ & -0.0324456 & 0.741 \\
FBG (mg/dL) & 1.001163 & $0.994861-1.007506$ & 0.0011627 & 0.718 \\
HbA1c (\%) & 1.209262 & $0.8777259-1.037205$ & 0.1900104 & 0.219 \\
Cholesterol (mg/dL) & 0.9499563 & $0.9852334-1.147663$ & -0.0513393 & 0.002 \\
Triglyceride (mg/dL) & 1.014751 & $1.002837-1.026806$ & 0.0146429 & 0.015 \\
HDL-C (mg/dL) & 1.063351 & $0.9201851-0.9806906$ & 0.0614255 & 0.115 \\
LDL-C (mg/dL) & 1.060507 & $0.798459-1.173723$ & 0.0587475 & 0.003 \\
VLDL-C (mg/dL) & 0.9541392 & $0.8499264-1.72052$ & -0.0469457 & 0.270 \\
\hline
\end{tabular}

(Abbreviations: CI, confidence interval; DM, diabetes mellitus; SBP, systolic blood pressure; DBP, diastolic blood pressure; BMI, body mass index; FBG, fasting blood glucose; HbAlc, glycosylated hemoglobin; HDL-C high density lipoprotein-cholesterol; LDL-C, low density lipoprotein-cholesterol; VLDL-C, very low density lipoprotein-cholesterol).

thy (P value of $0.001,0.000,0.002,0.015$, and 0.003 ). Regarding nephropathy and neuropathy, duration of diabetes was strongly associated with development of these complications ( $\mathrm{P}$ value $=0.000$ for each).

Table 7 shows that increasing age was a risk factor for developing CAD in patient with T2DM (P value 0.041). FBG was strongly associated for developing CVD in these patients ( $P$ value 0.042 ).

\section{Discussion}

About 30\% - 45\% of all patients of T2DM suffer from microvascular complications. People with DM have a four-fold greater risk of having a cardiovascular event, 2-to 4-fold greater risk of stroke and 15 times more likely to have lower limb amputation than people without DM [13].

In the present study, nephropathy was found in $56 \%$ of cases. Previous studies have reported prevalence of nephropathy in India as: 41\% [1], 30.2\% [14], 25.5\% [15], 36.3\% [16], and $23.0 \%$ [17], respectively. The WHO multicentric study of vascular disease in diabetics, reported a wide geographical variation in prevalence of diabetic nephropathy. It ranged from 2.4\% (Hong Kong), 23\% (Delhi), to $37 \%$ (Oklahoma, USA) respectively, [18] suggesting a visible variation in prevalence of nephropathy depending on ethnicity and population under study.

Higher prevalence of nephropathy (56\%) in our study is both due to the fact that the Indian ethnicity and the study being a hospital based. On applying multiple logistic regression analysis, we found a significant association of duration of DM with diabetic nephropathy (Table 6). Significant association of duration of $\mathrm{DM}$ and nephropathy have also been reported in previous Indian population 
Table 7. Results of multiple logistic regression analysis showing association of various risk factors with macrovascular complications. (a) Cerebro vascular disease; (b) Coronary artery disease.

(a)

\begin{tabular}{ccccc}
\hline Variable & Odds Ratio & $95 \% \mathrm{Cl}$ & Regression coefficient $\beta$ & P value \\
\hline Age (Years) & 1.023978 & $0.9832345-1.066409$ & 0.0236948 & 0.253 \\
Duration of DM (Years) & 0.9692579 & $0.8830328-1.063903$ & -0.0312245 & 0.511 \\
SBP (mm Hg) & 1.0266 & $0.9971288-1.056941$ & 0.026252 & 0.077 \\
DBP (mm Hg) & 1.017734 & $0.9385662-1.103579$ & 0.0175782 & 0.671 \\
BMI (Kg/m²) & 1.001836 & $0.8681151-1.156154$ & 0.0018339 & 0.980 \\
FBG (mg/dL) & 1.005517 & $1.000208-1.010854$ & 0.0055014 & 0.042 \\
HbAlc (\%) & 1.063118 & $0.8078992-1.398963$ & 0.0612065 & 0.662 \\
Cholesterol (mg/dL) & 1.007109 & $0.9872851-1.027331$ & 0.0070838 & 0.485 \\
Triglyceride (mg/dL) & 1.003872 & $0.9945877-1.013244$ & 0.003865 & 0.415 \\
HDL-C (mg/dL) & 1.037312 & $0.9830227-1.094599$ & 0.0366324 & 0.182 \\
LDL-C (mg/dL) & 0.9981724 & $0.9755718-1.021297$ & -0.0018293 & 0.876 \\
VLDL-C (mg/dL) & 0.9649452 & $0.9044688-1.029465$ & -0.035684 & 0.280 \\
\hline
\end{tabular}

(b)

\begin{tabular}{ccccc}
\hline Variable & Odds Ratio & $95 \% \mathrm{Cl}$ & Regression coefficient $\beta$ & P value \\
\hline $\begin{array}{c}\text { Age (Years) } \\
\text { Duration of DM }\end{array}$ & 1.056582 & $1.002316-1.113786$ & 0.055392 & 0.041 \\
$\quad 1.033227$ & $0.9264394-1.152323$ & 0.0326868 & 0.557 \\
SBP (mm Hg) & 0.992804 & $0.9564622-1030527$ & -0.007333 & 0.704 \\
DBP (mm Hg) & 0.9767028 & $0.8890805-1.07296$ & -0.0235729 & 0.623 \\
BMI (Kg/m²) & 1.144335 & $0.9517975-1.37582$ & 0.1348233 & 0.151 \\
FBG (mg/dL) & 0.9973596 & $0.9894435-1.005339$ & -0.0026439 & 0.516 \\
HbAlc & 1.140005 & $0.7764706-1.673742$ & 0.1310326 & 0.504 \\
Cholesterol (mg/dL) & 0.9977098 & $0.9736308-1.022384$ & -0.0022928 & 0.854 \\
Triglyceride (mg/dL) & 1.006625 & $0.9954544-1.017922$ & 0.0066035 & 0.246 \\
HDL-C (mg/dL) & 1.040883 & $0.9727079-1.113837$ & 0.0400696 & 0.246 \\
LDL-C (mg/dL) & 1.002423 & $0.972505-1.033262$ & 0.0024205 & 0.876 \\
VLDL-C (mg/dL) & 0.9721942 & $0.8987329-1.05166$ & -0.0281997 & 0.482 \\
\hline
\end{tabular}

(Abbreviations: CI, confidence interval; DM, diabetes mellitus; SBP, systolic blood pressure; DBP, diastolic blood pressure; BMI, body mass index; FBG, fasting blood glucose; HbAlc, glycosylated hemoglobin; HDL-C, high density lipoprotein-cholesterol; LDL-C, low density lipoprotein-cholesterol; VLDL-C, very low density lipoprotein-cholesterol).

studies [14] [16].

Diabetic neuropathy is one of the most common long-term microvascular complication of DM. In our study, neuropathy was present in $20 \%$ patients. Similar prevalence was also reported from South India i.e. 19.5\% [19]. The prevalence of neuropathy in earlier studies from India are 15.3\% [01], 26.8\% [14], and 
$33.1 \%$ [15] respectively. This variation may be due to glycemic control, genetic susceptibility to develop neuropathy and environmental factors. Statistical evaluation applying multiple logistic regression analysis for diabetic neuropathy, a positive association was found for duration of diabetes (Table 6). Agrawal et al. have published similar observation in their study [14].

In the present study retinopathy was found in $17.3 \%$ cases which is on par with the observations of earlier Indian studies i.e. 18\% [20], and 21.2\% [01] respectively. On the contrary, few other studies reported higher prevalences i.e. $32.5 \%$ [14] and 37.9\% [15] respectively, probably due to clinic based studies. Multiple logistic regression analysis for diabetic retinopathy revealed a positive association for age of patients, duration of diabetes, serum cholesterol, serum TG and serum LDL-C level (Table 6). Agarwal et al. found a positive association for age of patients, duration of diabetes, blood pressure, $\mathrm{FBG}$, and $\mathrm{HbA}_{1} \mathrm{C}$ with diabetic retinopathy [14].

The prevalence of cerebrovascular disease was $31.3 \%$ in the present study. The prevalence of CVD as reported in earlier studies varies from $0.5 \%$ to $9.2 \%$ amongst T2DM subjects in India [21]. Our own study from Cuttack in 2011 showed the prevalence of DM was $38.7 \%$ among stroke patients [22]. Similar observation was reported by Nagaraja D et al. from Bangalore i.e. 23.1\% [23]. This present study found that cerebral infarction was more common than haemorrhage ( $25 \%$ vs. $06 \%$ of total patients) which is at par with observation by Padma MV et al. (cerebral infaction 21.1\%, cerebral haemorrhage 6.35\%) [24].

Earlier studies by us from this centre (Cuttack) and Chennai had shown that at any given age subjects with DM had higher value of carotid intimal-medial thickness than non DM counterparts where the difference reached statistical significance after the age of 50 years [22] [25]. This is significant as the mean age of the patients suffering from CVD was 61.9 years in this study which can be extrapolated into corroborating the mean age of the patients with high prevalence of CVD. Multiple logistic regression analysis showed a positive association between FBG and CVD. Acute hyperglycemia could be one of the precipitating factor for CVD.

Coronary artery disease was found in $11.3 \%$ cases in our series. Prevalence of CAD was not uniform in different studies from India where earlier observations from Chennai revealed $11.4 \%$ [26], but the prevalence reported from North Delhi was $07 \%$ [01], where as a higher prevalence was reported from North West India i.e. 25.8\% [14] and recent studies from Chennai i.e. 21.4\% [2]. Prevalence of CAD in patients with DM was reported to be lower in this part of the country ${ }^{[27]}$. Multiple logistic regression analysis showed a definite association between age and CAD. Mohan V et al. have reported age and LDL-C as the risk factor for CAD [2].

Previous studies from this institute by us have shown, atherosclerosis to be more prevalent in subjects with DM and the quantum of involvement of vascular channels (coronary artery segments) were more profound as compared to the non-DM patients with CAD [28]. Such association suggests that uncontrolled 
hyperglycemia is an independent and important determinant for developing atherosclerotic vascular disease (ASVD) in subjects with DM [28] [29].

In one of our recent publication, it was shown that a significant inflammatory state was prevalent in patients with T2DM as compared to healthy individuals. Type 2 diabetes with macrovascular disease (MVD) had significantly higher high-sensitivity C-reactive protein (hs-CRP) as opposed to T2DM subjects without MVD. Further NF-kB expression was significantly higher where as adiponectin level was lower in patients of DM with MVD. Such proinflammatory state could be an independent determinant of atherosclerosis in this population. Further, this study has shown that persons with DM had significantly higher cholesterol and TG levels than non-DM counterparts [30].

Amongst acute metabolic complications, hyperglycemic hyperosmolar state (HHS) was found in $4 \%$ and DKA in $0.6 \%$ of cases respectively. Kitabchi AE et al. reported a prevalence of HHS to be less than $1 \%$ of all diabetes related hospital admissions and Faich GA et al. reported the prevalence of DKA to be $1.6 \%$ in Rhode Island [31] [32]. The high prevalence of HHS in our study could be due to hospital-based study.

In our study, it is observed that $44 \%$ patients have suffered from one or multiple infections. Most common infection was UTI (31.8\%). Most common pathogens isolated were E. coli $(61.9 \%)$ to be followed by $S$. aureus $(14 \%)$. Sterile culture was found in $19.4 \%$ of cases. Sridhar CB et al. reported symptomatic UTI to be $14 \%$ in mostly menopausal women with $\mathrm{DM}$, which is significantly lower than our observation, which may be due to the fact that our study is hospital based and most of the hospital admissions are due to infections that encompass UTI mostly [33]. Our observation is comparable with that of Bettegowda S et al. who reported prevalence of UTI to be $26.8 \%$ and $E$. coli was the most common pathogen isolated followed by Candida albicans and Jenifer J et al. from South India who reported prevalence of UTI to be $42.8 \%$ and $E$. coli as the most common pathogen isolated where as sterile culture was found in $24.3 \%$ cases [34] [35].

Pneumonia was found in $30.3 \%$ cases in our study which is higher than that reported by previous Indian studies i.e. 11.6\% [33] and 4.4\% [34] possibly because of a referral tertiary care hospital and emphasizes the importance of non-tubercular pulmonary infection in subjects with T2DM.

Tuberculosis (TB) was found in $4.5 \%$ cases in our series. Earlier study by Patel JC from India had reported TB to be the most common associated illness (5.9\%) with DM [36]. Other studies done much earlier by Bhatia (1975), Bahulkar (1975), Nanda and Tripathy (1968) had reported the prevalence of TB in DM to be $14 \%, 4.5 \%$ and $12 \%$ respectively [37]. The low prevalence in our study could be due to awareness and early detection of Pulmonary TB with wide availability of anti-tubercular drug therapy.

Diabetic foot was found to be present in $4.5 \%$ cases in our study. Viswanathan $\mathrm{V}$ et al. in a multicentric study from India reported the prevalence of diabetic foot infection to be $6 \%-11 \%$ [38]. Our observation is at par with the observation of Pendsey SP, who found the point prevalence of foot ulcers in patients of 
diabetes in clinic population to be $3 \%$ [39].

NAFLD was found in $16.6 \%$ patients. Recent studies from India have reported the prevalence of NAFLD as 56.5\% [40] and 57.2\% [41] respectively in T2DM patients. The low prevalence in our series compared to those published from metro cities may be likely due to lower prevalence of obesity in our population.

Out of 150 patients studied, 33 patients (18.6\% of total, male: $75.7 \%$, female: $24.3 \%)$ died due to various diabetes related complications. The mean age at death was 62.2 years (male: 62.6 years, female: 61.2 years). Das et al. reported the age at the time of death for people with DM to be 55 - 61 years and that mortality among hospitalized patients with non-insulin-dependent DM was nearly $20 \%$, which is comparable with the findings of present study [7]. A recent population based study from South India by Mohan et al. reported the mean age of death to be 66 years and mortality related to diabetic complications to be $11.9 \%$ [6]. Analysing the causes of mortality in our cohort it was found that CVD (36.4\%) was the most common cause of death followed by infection (27.3\%), CAD (15.1\%), CKD (12.1\%), and HHS (9.0\%). Cardiovascular (CV) related death (which includes CAD and CVD) was by far the most common cause of death in our series accounting for more than half $(51.5 \%)$ of total death which could be due to the fact that macrovascular complications occured earlier than microvascular complications. Mohan et al. also had similar observations from South India, reported CV related deaths was the most common etiology, accounting for mortality in nearly $53 \%$ cases [6]. Das et al. also had similar observation from this institution 25 years ago reporting CV related death to be $62.5 \%$ cases [7]. Bhansali et al. from a tertiary care hospital in North India reported CV related death as second most common cause of death (23.4\%) following infections [42]. Infections, which accounted for $27.3 \%$ cases of death was the second most common cause of death in our study. Das et al. also reported infection as the leading cause of death (33.7\%) in their study [7]. Bhansali et al. also observed infection as the most common cause of death $(46.5 \%)$ in their series [42]. The continued prevalence of infections in causing mortality in our patients may be attributed to overall high incidence of infections in this part of the country and poor glycemic control in these patients. Analysing the above figures of prevalence of infection and CV related death in T2DM patients in this part of the country, it seems that there is a shift in pattern of death from infection to CV related death possibly due to increased awareness among patients of DM regarding infection, seeking health service at right time, as well as availability of better health care facilities including ICU care. Chronic kidney disease (CKD) was the third most common cause of death in our study (12.1\%). Bhansali et al. also reported a prevalence of $9.4 \%$ of CKD related death [42]. Mohan et al. from South India reported CKD related death to be $23.5 \%$ [6]. This could be due to poor glycemic control, inadequate facility for renal replacement therapy (RRT) like dialysis/transplant, and lack of affordability to RRT. 


\section{Limitations of the Study}

As this was a tertiary hospital based study and may not be real reflection of the population. The work was a prospective cross-sectional study, it is not possible to determine whether levels of variables showing association with complications actually preceded the development of the complications. Hence, the clinical and laboratory variables showing association with the complications in this study may only be interpreted as potential risk factors. As this study was a part of Post Graduate thesis and so was time limited. More prolonged study is required in future.

\section{Conclusions}

This study highlights the high prevalence of vascular complications and infections in T2DM patients of this part of India. Nephropathy was the most common microvascular complication and cerebrovascular disease was the most common macrovascular complication. Macrovascular complications occurred earlier than microvascular complications. CV-related death was the most common cause of death (CVD more than CAD) followed by infections and CKD. CVD occurred earlier and was the most common cause of mortality in our patients of T2DM.

This study emphasizes the need for screening of all T2DM patients for complications at the time of diagnosis for early detection. The higher prevalence of cerebrovascular disease and nephropathy as observed by us may be similar to a few Indian studies but a visibly different from world literature. This suggests further work on etiopathogenesis of T2DM as well as its complications in our population group.

\section{Conflict of Interest}

This study was neither funded nor sponsored by any agency or pharmaceutical firm. This original research work is part of the regular postgraduate research thesis work of the Postgraduate Department of Medicine. The authors undertake that there is no conflict of interest, whatsoever with anyone.

\section{References}

[1] Chawla, R. (2012) Complications of Diabetes. Jaypee Brothers Medical Publishers, New Delhi, 1-53. https://doi.org/10.5005/jp/books/11685

[2] Mohan, V., Deepa, R. and Shanthirani, S. (2001) Prevalence of Coronary Artery Disease and Its Relationship to Lipids in a Selected Population in South India-The Chennai Urban Population Study (CUPS No 5). Journal of the American College of Cardiology, 38, 682-687. https://doi.org/10.1016/S0735-1097(01)01415-2

[3] Premlatha, G., Shanthirani, S. and Deepa, R. (2000) Prevalence and Risk Factors of Peripheral Vascular Disease in a Selected South Indian Population: The Chennai Urban Population Study. Diabetes Care, 23, 1295-1300. https://doi.org/10.2337/diacare.23.9.1295

[4] Schuetz, P., Castro, P. and Shapiro, N.I. (2011) Diabetes and Sepsis, Preclinical Findings and Clinical Relevance. Diabetes Care, 34, 771-778. 
https://doi.org/10.2337/dc10-1185

[5] United Nations (2009) World Population Prospects. The 2008 Revision.

[6] Mohan, V., Shantirani, C.S. and Deepa, M. (2006) Mortality Rates Due to Diabetes in a Selected Urban Study Indian Population: The Chennai Urban Population Study (CUPS 16). Journal of the Association of Physicians of India, 54, 113-117.

[7] Das, S., Mishra, R.K. and Jena, B.B. (1991) Mortality Events amongst Non-Insulin Dependent Diabetes Mellitus Patients in Orissa. Journal of the Association of Physicians of India, 39, 519-520.

[8] World Health Organization (1980) Expert Committee on Diabetes Mellitus Second Report. WHO Technical Report Series, Geneva.

[9] American Diabetes Association (2006) Position Statement: Standards of Medical Cars in Diabetes. Diabetes Care, Suppl. 2, S5-S42.

[10] National Kidney Foundation (2007) KDOQI Clinical Practice Guidelines and Clinical Practice Recommendations for Diabetes and Chronic Kidney Disease. American Journal of Kidney Diseases, 49, S42-S61.

[11] Mythili, A., Kumar, K.D., Subrahmanyam, K.A., Kenkateswarlu, K. and Butchi, R.G. (2010) A Comparative Study of Examination Score and Quantative Sensory Testing in Diagnosis of Diabetic Polyneuropathy. International Journal of Diabetes in Developing Countries, 30, 43-48. https://doi.org/10.4103/0973-3930.60007

[12] Das, S., Singh, S.P, Parida, P.K. and Mallik, R.N. (2010) Non-Alcoholic Fatty Liver Disease in Subjects with Type-2 Diabetes Mellitus and Non-Diabetics with Special Reference to Insulin Resistance, Hepatic Histopathological Changes. Diabetes \& Metabolic Syndrome: Clinical Research and Reviews, 4, 226-229.

[13] Cade, W.T. (2008) Diabetes-Related Microvascular and Macrovascular Diseases in the Physical Therapy Setting. Physical Therapy, 88, 1322-1335.

https://doi.org/10.2522/ptj.20080008

[14] Agarwal, R.P., Ola, V. and Bishnoi, P. (2014) Prevalence of Micro and Macrovascular Complications and Their Risk Factors in Type-2 Diabetes Mellitus. The Journal of the Association of Physicians of India, 62, 504-508.

[15] Pradeepa, R., Prabhu, A.K. and Jebarani, S. (2011) Use of Large Diabetes Electronic Medical Record System in India: Clinical and Research Applications. Journal of Diabetes Science and Technology, 5, 543-552. https://doi.org/10.1177/193229681100500309

[16] Varghese, A., Deepa, R. and Reema, M. (2001) Prevalence of Microalbuminuria in Type-2 Diabetes Mellitus at a Diabetes Centre in South India. Postgraduate Medical Journal, 77, 399-402. https://doi.org/10.1136/pmj.77.908.399

[17] Yajnik, C.S., Naik, S.S. and Rout, K.N. (1992) Urinary Albumin Excretion Ratio (AER) in Newly Diagnosed Type 2 Indian Diabetic Patients Is Associated with Central Obesity and Hyperglycemia. Diabetes Research and Clinical Practice, 17, 55-60.

[18] Diabetes Drafting Group (1985) Prevalence of Small Vessel and Large Vessel Disease in Diabetic Patients from 14-Centres. The World Health Organisation Multinational Study of Vascular Disease in Diabetics. Diabetologia, 28, 615-640. https://doi.org/10.1007/BF00290267

[19] Ashok, S., Ramu, M. and Deepa, R. (2002) Prevalence of Neuropathy in Type 2 Diabetic Patients Attending a Diabetes Centre in South India. Journal of the Association of Physicians of India, 50, 546-550.

[20] Raman, R., Rani, P.K. and Reddi Rachepalle, S. (2009) Prevalence of Diabetic Retinopathy in India: Sankar Nethralaya Diabetic Retinopathy Epidemiology and Molecular Genetics Study Report 2. Ophthalmology, 116, 311-318. 
[21] Das, S. (1993) Cerebrovascular Complications in NIDDM. Journal of the Association of Physicians of India, 41, 57-65.

[22] Das, S., Chakravorty, K. and Patnaik, M. (2011) The Relationship of Carotid Plaque, Intima Media Thickness (IMT), Resistivity Index (RI) and Pulsatility Index (PI) in Asian-Indian Patients with Acute Ischemic Stroke with and without Type-2 DM. International Journal of Clinical Medicine, 2, 561-566. https://doi.org/10.4236/ijcm.2011.25092

[23] Nagaraja, D., Gururaj, G. and Girish, N. (2009) Feasibility Study of Stroke Surveillance: Data from Bangalore, India. Indian Journal of Medical Research, 130, 396-406.

[24] Padma, M.V. and Bajaj, J.S. (1999) Diabetes and Stroke. In: Bansal, B.C., Ed., Recent Concepts in Stroke, The Association of Physicians of India, Mumbai, 79-95.

[25] Mohan, V., Ravikumar, R. and Shanthirani, S. (2000) Intimal Medial Thickness of the Carotid Artery in South Indian Diabetic and Non Diabetic Subjects: The Chennai Urban Population Study (CUPS). Diabetologia, 43, 494-499.

https://doi.org/10.1007/s001250051334

[26] Ramachandran, A., Snehalatha, C. and Satyavani, K. (1999) Prevalence of Vascular Complications and Their Risk Factors in Type 2 Diabetes. Journal of the Association of Physicians of India, 47, 1152-1156.

[27] Das, S. and Behera, M. (2015) Coronary Artery Disease in Diabetes. World Clinics. Diabetology, 1, 173-191.

[28] Mishra, T.K., Das, S. and Patnaik, U.K. (2004) Relationship of Metabolic Syndrome with Quantum of Coronary Artery Disease in Indian Patients with Chronic Stable Angina. Metabolic Syndrome and Related Disorders, 2, 187-191.

https://doi.org/10.1089/met.2004.2.187

[29] Das, S., Dash, G.K. and Behera, M. (2007) Prevalence of Metobolic Syndrome in Angiographically Established Coronary Artery Disease Patients. South Asian Journal of Preventive Cardiology, 11, 93-99.

[30] Misra, D.P., Das, S. and Sahu, P.K. (2012) Prevalence of Inflammatory Markers High-Sensitivity C-Reactive Protein, Nuclear Factor B, and Adipanectin in Indian Patients with Type 2 Diabetes Mellitus with and without Macrovascular Complications. Metabolic Syndrome and Related Disorders, 10, 209-213. https://doi.org/10.1089/met.2011.0044

[31] Kitabchi, A.E., Umpierrez, G.E. and Murphy, M.B. (2001) Management of Hyperglycemic Crisis in Patients with Diabetes. Diabetes Care, 24, 131-153. https://doi.org/10.2337/diacare.24.1.131

[32] Faich, G.A., Fishbein, H.A. and Ellis, S.E. (1983) The Epidemiology of Diabetic Acidosis: A Population Based Study. American Journal of Epidemiology, 117, 551558. https://doi.org/10.1093/oxfordjournals.aje.a113577

[33] Sridhar, C.B., Anjana, S. and Thomas, M.J. (2002) Acute Infections. In: Tripathy, B.B., Das, A.K., Madhu, S.B. and Mohan, V., Eds., RSSDI Text Book of Diabetes Mellitus, Jaypee Publishers, 471-477.

[34] Bettegowda, S., Iyengar, V.S. and Gosain, V. (2014) Clinical Profile and Spectrum of Infections in Type 2 Diabetes Mellitus Patients: A Retrospective Study from Rural Tertiary Care Hospital of South Karnataka, India. Scholars Journal of Applied Medical Sciences, 2, 3331-3336. https://doi.org/10.4103/0971-4065.57107

[35] Jenifer, J., Geethalakshmi, S., Satyavani, K. and Viswanathan, V. (2009) Prevalence of Lower Urinary Tract Infection in South Indian Type 2 Diabetic Subjects. Indian Journal of Nephrology, 19, 107-111. 
[36] Patel, J.C. (1989) Complications in 8793 Cases of Diabetes Mellitus 14 Years Study in Bombay Hospital, Bombay, India. Indian Journal of Medical Sciences, 43, 177-183.

[37] Samar, B. (1998) Endocrine \& Metabolic Aspects in Tuberculosis. Update on Tuberculosis. API, WB Branch, 25-29.

[38] Viswanathan, V., Thomas, N. and Tandon, N. (2005) Profile of Diabetic Foot Complications and Its Associated Complications-A Multicentric Study from India. Journal of the Association of Physicians of India, 53, 933-936.

[39] Pendsey, S.P. (1994) Epidemiological Aspects of Diabetic Foot. International Journal of Diabetes in Developing Countries, 14, 37-38.

[40] Kalra, S., Vithalani, M. and Gulati, G. (2013) Study of Prevalence of Nonalcoholic Fatty Liver Disease (NAFLD) in Type 2 Diabetes Patients in India (SPRINT). Journal of the Association of Physicians of India, 61, 448-453.

[41] Agarwal, A.K., Jain, V. and Baruah, B.P. (2011) Prevalence of Nonalcoholic Fatty Liver Disease and Its Correlation with Coronary Risk Factor in Patients with Type 2 Diabetes. Journal of the Association of Physicians of India, 59, 351-354.

[42] Bhansali, A., Chattopadhyaya, A. and Dash, R.J. (2003) Mortality in Diabetes: A Retrospective Analysis from a Tertiary Care Hospital in North India. Diabetes Research and Clinical Practice, 60, 119-124. 


\section{Abbreviations}

AER: Albumin excretion rate

BMI: Body Mass Index

CAD : Caronary Artery Disease

CKD: Chronic Kidney Disease

CUPS: Chennai Urban Population Study

CV: Cardio Vascular

CVD: Cerebro Vascular Disease

DM: Diabetes Mellitus

DKA: Diabetic Keto Acidosis

HbAIC: Glycosylated Haemoglobin

HDL-C: High-density Lipoprotein-Cholesterol

HHS: Hyperglycemic Hyperosmolar State

ICH: Intracerebral Haemorrhage

LDL-C: Low-density Lipoprotein Cholesterol

MVD: Macro Vascular Disease

NF-kB: Nuclear factor-kappa B

NPDR: Non-Proliferative Diabetic Retinopathy

NAFLD: Non Alcoholic Fatty Liver Disease

PVD: Peripheral Vascular Disease

TB: Tuberculosis

T2DM: Type 2 Diabetes Mellitus

TG: Triglyceride

UTI: Urinary Tract Infection

Submit or recommend next manuscript to SCIRP and we will provide best service for you:

Accepting pre-submission inquiries through Email, Facebook, LinkedIn, Twitter, etc. A wide selection of journals (inclusive of 9 subjects, more than 200 journals)

Providing 24-hour high-quality service

User-friendly online submission system

Fair and swift peer-review system

Efficient typesetting and proofreading procedure

Display of the result of downloads and visits, as well as the number of cited articles

Maximum dissemination of your research work

Submit your manuscript at: http://papersubmission.scirp.org/

Or contact jdm@scirp.org 